We thank Dr J Costello and the staff of the chest unit at this hospital for their help with this study.

1 Recchione C, Galante E, Secreto G, Cavalleri A, Dati V. Abnormal serum hormone levels in lung cancer. Tumori 1983;69:293-8.

2 Chaudhuri PK, Thomas PA, Walker MJ, Briele HA, Das Gupta TK, Beattie CW. Steroid receptors in human lung cancer cytosols. Cancer Lett 1982;16:327-32.

3 Iqbal MJ, Johnson MW. Study of steroid protein binding by a novel "two-tier" column employing Cibacron blue F3GA-Sepharose 4B sex hormone binding globulin. F Steroid Biochem 1977;8: 977-83.

4 Iqbal MJ, Dalton M, Sawers RS. Binding of testosterone and oestradiol to sex steroid binding globulin, human serum albumin and other plasma proteins: evidence for non-specific binding of oestradiol to sex hormone binding globulin. Clin Sci 1983;64:307-14.

5 Chlebowski RT, Heber D. Hypogonadism in male patients with metastatic cancer prior to chemotherapy. Cancer Res 1982;42:2495-8.

Accepted 3 July 1986

Liver Unit, King's College Hospital and School of Medicine and Dentistry, London SE5 8RX

TIM P CORBISHLEY, BSC, research assistant

JOHN J KEATING, MRCP, research fellow

PHILIP J JOHNSON, MRCP, senior lecturer

ROGER WILLIAMS, MD, FRCP, director and consultant physician

Correspondence to: Dr Williams

\section{Effect of passive exposure to smoking on age at natural menopause}

Several studies have shown that women who smoke cigarettes reach the menopause sooner than those who do not, irrespective of potential confounding factors including marital state, parity, and body weight. ${ }^{1}$ The reason for this is unknown, but it may be due to the destruction of irreplaceable primary oocytes by benzo(a)pyrene and other toxic substances in cigarette smoke. ${ }^{2}$ Because exposure to toxic agents in tobacco smoke may also occur from passive smoking (including transplacental exposure ${ }^{3}$ and inhalation of ambient tobacco smoke) we investigated associations between passive exposure to smoking and age at natural menopause.

\section{Subjects, methods, and results}

We studied 261 women aged 35 and over who had acted as controls in a previous case-control study. ${ }^{4}$ Data were analysed with the Kaplan-Meier procedure and conditional logistic regression' by methods analogous to life table analysis of survival data in a clinical trial or cohort study. In the life tables "survival" was equated with remaining premenopausal, "death" with the onset of menopause, and "withdrawal" with being interviewed before menopause or reporting a non-natural menopause.

The Kaplan-Meier estimate of mean age at natural menopause was $49 \cdot 7$ years for smokers and 50.8 years for non-smokers. The mean ages in non-smokers whose spouses also did not smoke and non-smokers whose spouses smoked were 51.9 and 49.8 years respectively (figure).

In a model comparing active smokers with non-smokers by conditional logistic regression only a modest increase in the risk of early menopause (odds ratio (OR) 1.3; approximate $95 \%$ confidence interval (CI) $0 \cdot 8$ to $2 \cdot 2$ ) was shown. Much stronger trends were evident when subjects who reported no exposure to active or passive smoking were compared with subjects exposed to either only passive smoking (OR 1.9; CI 1.0 to 3.9) or to active smoking (OR 1.9; CI 0.9 to 3.7 ). Race, education, year of birth, and alcohol intake were not significant independent indicators of the risk of early menopause; including them as covariates increased the estimates of risk associated with both active (OR 2.3; CI 1.1 to 4.9 ) and passive (OR $2 \cdot 1$; CI $1 \cdot 0$ to $4 \cdot 5)$ smoking. Early exposure of a woman to smoking by her father was not associated with a risk of early natural menopause (OR $1 \cdot 1$, CI 0.6 to 1.9 ). The mean age at menopause was $2 \cdot 2$ years younger among women whose mothers smoked, but only four subjects were in this group.

\section{Comment}

In previous studies of the association between exposure to smoking and age at menopause non-smokers included subjects who might have been passively exposed to smoking. Our study, though much smaller, refined the classification of subjects by omitting passive smokers from the group of nonsmokers. This reclassification increased the difference in ages at menopause from $1 \cdot 1$ to $2 \cdot 2$ years and increased estimates of the risk of early menopause associated with active smoking in our data from $1 \cdot 3$ to $1 \cdot 9$. These differences show the importance of distinguishing non-smokers who are passively exposed from those who are not.

Our data also suggest that early natural menopause is associated with passive smoking. The increase in risk of early menopause observed for passive smoking was of similar magnitude to that observed for active smoking, but the study population was small and the true magnitude of

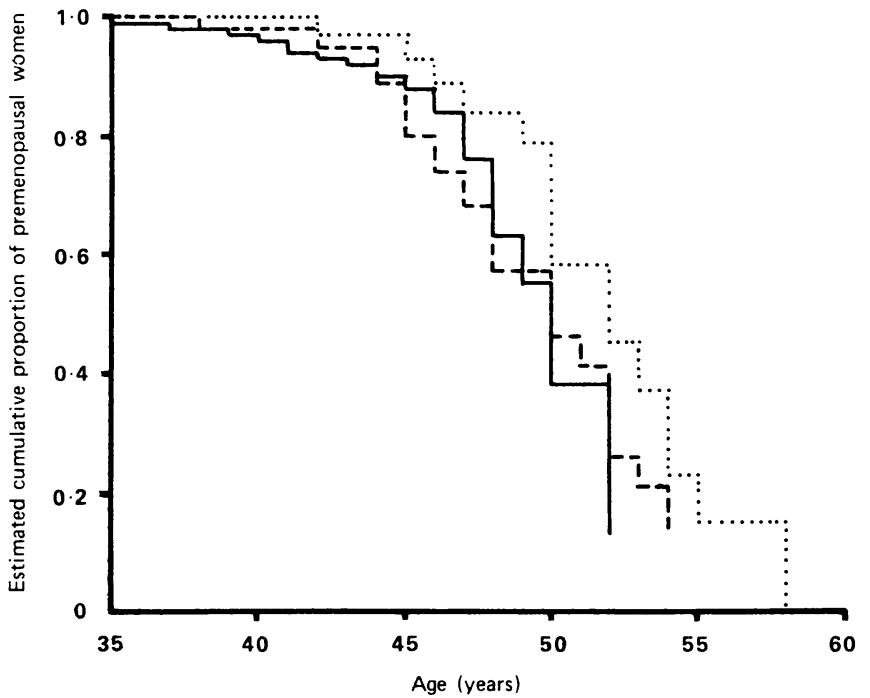

Kaplan-Meier estimates of cumulative proportion of women premenopausal by age for non-smokers who are not exposed passively to smoking (...); nonsmokers who are exposed passsively to smoking (_- _ ) ; and active smokers $(\longrightarrow)$.

either effect is uncertain. The findings thus suggest that there is an effect from passive smoking but do not justify the conclusion that effects of active and passive exposure are equivalent. Further studies of larger populations are needed to confirm and further quantify these associations, consider the effects of additional potentially confounding variables (such as relative body weight) not studied here, and investigate possible transplacental effects.

We thank Dr Barbara S Hulka, department of epidemiology, University of North Carolina School of Public Health, for providing helpful advice at several stages of this study; Dr Jay H Lubin, environmental epidemiology branch, National Cancer Institute, for the computer program used for conditional logistic regression analysis; and Karen L Milne for helping in data management and analysis.

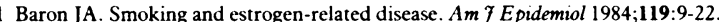

2 Mattison DR, Thorgeirsson SS. Smoking and industrial pollution, and their effects on menopause and ovarian cancer. Lancet 1978;i:187-8.

3 Everson RB. Individuals transplacentally exposed to maternal smoking may be at increased cancer risk in adult life. Lancet $1980 ; \mathrm{ii}: 123-7$.

4 Sandler DP, Everson RB, Wilcox AJ, Browder JP. Cancer risk in adulthood from early life exposure to parents' smoking. Am f Public Health 1985; 75:487-92.

5 Gail MH, Lubin JH, Rubinstein LV. Likelihood calculations for matched case-control studies and survival studies with tied death times. Biometrika $1981 ; 68: 703-7$.

(Accepted 7 July 1986)

Epidemiology Branch and Statistics and Biomathematics Branch, National Institute of the Environmental Health Sciences, PO Box 12233, Research Triangle Park, North Carolina 27709, USA

RICHARD B EVERSON, MD, MPH, medical officer

DALE P SANDLER, PHD, epidemiologist

ALLEN J WILCOX, MD, PHD, medical officer

DINA SCHREINEMACHERS, $\mathrm{Ms}$, technical staff

DAVID L SHORE, MS, research assistant

CLARICE WEINBERG, PHD, statistician

Correspondence to: Dr Everson, Epidemiology Branch. 